bichromate solution may not be very accurately determined with silver.

Iowa College, Grinveli, Iowa,

March 21, I903.

\title{
REDUCIBILITY OF SOME METALLIC OXIDES BY HYDRO- GEN AND CARBON MONOXIDE.
}

\author{
By IRving W. FAY and ALBERT F, SEEkER.
}

Received April II, I903.

Ix passing hydrogen gas over silver oxide in a bulb tube to show the reduction of the oxide to metallic silver I frequently noticed slight explosions and a sudden change of oxide to metal at what seemed a low temperature for such an effect. In the literature, I found that silver oxide was reduced by hydrogen at $85^{\circ 1}$ and $100^{\circ}$. Wishing to test the accuracy of these temperatures, the following investigation was begun with the impression that both temperatures were too high. Both hydrogen and carbon monoxide were used to reduce the silver oxide. Later, other oxides were submitted to the reducing action of these two gases.

The method used by Mueller ${ }^{2}$ consisted in placing a glass tube containing the oxide in the sand of a sand-bath. A thermometer was thrust into the sand so that the bulb was close to the part of the tube containing the oxide. The tube was bent at a right angle, one end sealed, the open lower end dipping under water. The tube was filled with hydrogen and the temperature increased. Reduction of the oxide was shown by ascent of water in the open end or arm of the tube.

Wright ${ }^{3}$ and Luff placed the metallic oxides for reduction in a test-tube arranged vertically in a paraffin bath, about 0.5 gram being taken for each observation. The mouth of the test-tube was closed by a cork with three perforations, one for the thermometer, the bulb of which reached to the bottom of the tube, one for the entrance tube for leading in the pure gas, and the third for an exit tube just passing through the cork and bent at a right angle and coupled by a rubber joint to a Will and Varrentrapp's ammonia

1 wöhler: Ann. Chem. (Liebig), 30, 4.

2 Miuller: Pogg. Ann., 136, 5 t.

$3 \mathrm{~J}$. Chem. Soc. (London), 33, . 
buib apparatus filled with clear baryta water. In order to make sure that the entering gas was at the same temperature as the bath, the entrance tube was bent over, lownwards and upwards, so as to form a $\mathrm{L}$, reaching down to the bottom of the test-tube and lying close to the outer surface; the upper part of the tube was wrapped with lamp-wick to prevent aerial cooling. Loss of weight was used to determine actual reduction, and fifteen minutes was the time allowed for each test.

EXPERIMINATAL PART.

REDUCTION OF SILVER OXIDE BY HYDROGEN.

The oxide was that sold as chemically pure. As a preliminary test, silver oxide was placed in a tube about 0.25 inch internal diameter, so bent that it was easily clamped in position with the bend I inch below the surface of oil in an oil-bath. A thermometer was hung in the oil with its bulb close to the bend of the glass tube where the silver oxide lay. Hydrogen gas from zinc and hydrochloric acid without drying was passecl slowly over the oxide. At a temperature of $100^{\circ}$, the oxide was reduced in a few minutes to the familiar grayish white powder of metallic silver. An hour's heating at $80^{\circ}$ did not seem to reduce any oxide, but examination under a microscope showed metallic silver in the dark powder. At $40^{\circ}$ the microscope revealed particles of silver. The silver oxide had previously been examined under the microscope, and metallic silver proved absent. Below $40^{\circ}$ no reduction was apparent, but, suspecting that longer exposure to hydrogen would show a reduction, a quantity of oxide was sealed in an atmosphere of hydrogen within a tube and laid aside in a drawer at ordinary laboratory temperature. At the end of a week, the black color of the oxide was completely changed to white metallic silver, and moisture could be seen upon the glass inside of the tube. A similar tube similarly charged was placed alongside a block of ice in a refrigerator. In a week, the powder had become blacker, and moisture was shown by the powder sticking to the tube, and metallic silver was seen under the microscope.

To insure the constant maintenance of $0^{\circ} \mathrm{C}$. during the test, a wooden $\mathrm{V}$-shaped trough was used, I foot in length and about the same in height. The tube containing the oxide lay along the 
bottom of the trough, the end projecting through a round hole so bored as to allow of easy removal and insertion of the oxide tube. Crushed ice was kept in the trough, and woolen coverings were placed over it.

Pure dry hydrogen gas was used for the subsequent work. It was made from zinc and sulphuric acid, to which some copper sulphate solution had been added, and it was further purified by passing it in succession through two wash-bottles containing permanganate of potash solution, a U-tube containing pumice saturated with a solution of mercuric chloride, a U-tube filled with pumice wet with a solution of potassium hydroxide, a piece of iron pipe, containing copper tacks and copper gauze, heated to redness (the wrapped ends kept cool by water dripping from capillary tubes), a U-tube containing anhydrous copper sulphate, a tower of calcium chloride, and a wash-bottle half-filled with phosphorus pentoxide. The rubber joints were covered with shellac varnish.

A glass tube 0.5 inch internal diameter was charged with silver oxide, and capillary tubes were drawn out at each end. The tube was placed in the ice trough and, when some time had elapsed to allow it to attain $0^{\circ} \mathrm{C}$., a stream of hydrogen was passed for one hour, and then a manometer tube consisting of a capillary tube of $U$-shape half-filled with mercury and so bent that the parallel arms were but an inch apart, the height of the manometer being 12 inches, was joined to the oxide tube by a short, tightly-fitting black rubber tubing, afterward covered with shellac varnish.

The capillary where the hydrogen had entered was sealed, and except for additions of ice from time to time, the apparatus was left to itself for five days. The progress of the reduction is shown in the following table of corrected readings.

Rise of mercury in the arm toward the oxide tube:

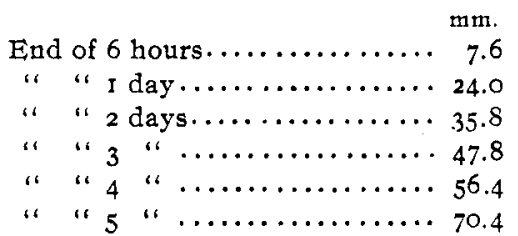

On removing the tube from the melting ice, moisture was plainly visible condensed on the upper side.

The amount of reduction was so much more than was expected 
that it was feared hydrogen had diffused outward through the rubber joint, and thereby increased the apparent reduction. Another tube was prepared to test this by having the oxide tube and the capillary manometer entirely of one piece of glass. A long capillary tube was drawn out from the end of an 8-inch glass tube; it was then bent at a right angle and turned in $\mathrm{C}$-shape, the parallel arms close together; another very short capillary was drawn out close to where the manometer capillary joinecl the main tube; this short second capillary on the same end as the manometer allowed a continuous stream of hydrogen to pass without disturbance of the mercury in the manometer. This tube was charged along the bottom with silver oxide, the open end drawn out to a capillary and then placed in the ice trough to acquire $o^{\circ}$.

A stream of pure clry hydrogen was passed for one hour, and several times the tube was rocked in such a manner as to bring the mercury close up to the short capillary, through which hyctrogen was passing. This accomplished the expulsion of all air in the branch of the manometer tube next the oxide. The capillarics at both ends were sealed and the tube maintained at $o^{\circ}$ for twelve days by an abundance of ice and careful covering.

The amount of reduction day by day was as follows:

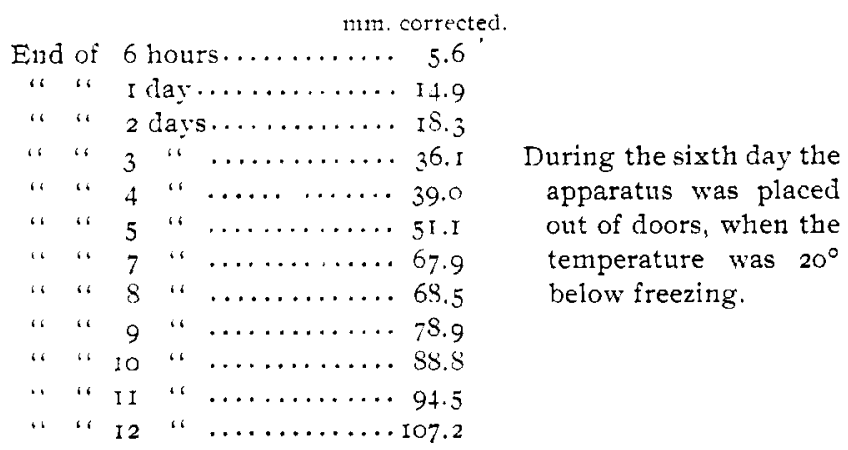

Auric oxide, subjected to exactly similar treatment with hydrogen gas at $0^{\circ}$, showed a more rapid reduction than silver oxide, as the following table will show.

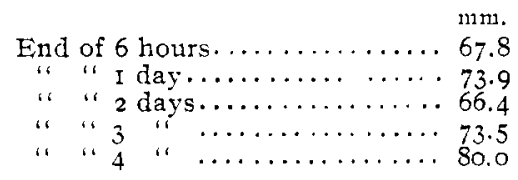


In all of these tests the surface-tension of the mercury in the capillary manometer was such that movement was sluggish, and the tube needed frequent tapping to cause a readjustment of the mercury column.

REDUCTION OF THE OXIDES OF MERCURY BY HYDROGEN.

The oxide was placed in a glass tube 0.25 inch inside diameter, bent at such an angle that the bend itself was I.5 inches below the surface of oil in an oil-bath, in which it was heated. At first, on passing the hydrogen, the temperature was rapidly increased until the oxide could clearly be seen to exhibit reduction. A thermometer was hung in the bath with its bulb close to the bend of the tube where the oxide lay. To determine the lowest temperature at which six hours' contact with hydrogen would show reduction, tests were made with fresh portions of oxide at each interval of $5^{\circ}$ below the temperature discovered in the above process, and reduction proved by the vacuum formed within the tube. Each test was as follows: The ends of a glass tube 0.5 inch in diameter and 6 inches long were drawn out to capillary tubes 3 inches long. The oxide was introduced and spread along the bottom of the tube and a large excess of pure hydrogen passed through. Both capillaries were sealed and bent upward to allow of immersion in an oil-bath of moderate size. A constant temperature was maintained by a thermostat, and careful protection of the flame and bath from drafts. Any reduction at the end of the six hours' heating revealed itself by a partial vacuum formed within the tube when cooled to its original temperature.

To ascertain the presence of a slight vacuum and therefore of the slightest reduction of the oxide, a manometer was used, consisting of two capillary glass tubes fastened side by side to a millimeter scale, their lower ends joined by rubber tubing. The oxide-tube to be examined was connected by a small rubber tube to one arm of the manometer, $i$. e., the capillary tube, scratched with a file near the tip, was thrust into the rubber tube, the other end of which was joined to one arm of the manometer. The mercury in both arms was brought to a level, the tip of the oxide tube broken off within the rubber tube, and any partial vacuum was at once revealed by a rise of the mercury in the arm of the manometer next to the oxide-tube. Blank tests made at different times showed 
the largest error due to manipulation to be $5 \mathrm{~mm}$. No metallic oxide was regarded as showing reduction unless the corrected vacuum attained exceeded $5 \mathrm{~mm}$. The lowest temperatures for the reduction of the oxides of mercury are as follows:

Mercurous oxide.................. $80^{\circ} \mathrm{C}$.

Yellow mercuric oxide............... $50^{\circ} \mathrm{C}$, at $\mathrm{r} 30^{\circ} \mathrm{C} .{ }^{1}$

Red mercuric oxide.................. $115^{\circ} \mathrm{C}$., at $230^{\circ} 1$

REDLCTION OF SOME METALLIC OXIDES BY CARBON MONOXIDE.

The method consisted in passing pure dry carbon monoxide over the metallic oxide charged in a bent glass with the bend immersed I.5 inches below the surface of the oil in an oil-bath. The thermometer bulb was fixed close against the bend of the immersed tube where the oxide lay. A constant temperature at any degree was secured by a thermostat, and protection of the flame and oilbath from clrafts. Any carbon dioxide formed by reduction of the metallic oxicle was detected by passing the gas through a Marchand tube, containing a solution of barium hydroxide.

The carbon monoxide was made from coarsely crushed potassium ferrocyanide and concentrated sulphuric acid. Raising the temperature rapidly until evolution of the gas began and then lowering the flame, gave perfect control of the gas, which otherwise may be given off tumultuously. The gas was purified and dried by passing it through a train consisting of two wash-bottles of solutions of sodium hydroxide and one of lime-water, a tower of calcium chloride, and a wash-bottle half-filled with phosphorus pentoxide. The barium hydroxide solution, one-third the strength of saturation, was shaken with barium carbonate to increase its sensitiveness to carbon dioxide. This solution was found sensitive to the carbon dioxide in 0.25 liter of atmospheric air, which is $0.075 \mathrm{cc}$. Lime-water was much less sensitive, requiring 0.75 liter of atthospheric air to give an equal amount of turbidity in the same volume of liquid. Efficiency of the purifying train was proved by making blank tests from time to time. The Marchand tube containing the solution of barium hydroxide was protected from backward diffusion of carbon dioxide from the air bu a second Marchand tube, charged like the first. Each test was continued for six hours, the gas passing at the rate of one to two

1 Miller: Pogg. Ann., 136, 52 . 
bubbles per second, the temperature kept constant during the time. A first test was made sufficiently high to find where reduction actually took place with rapidity, then successive tests at intervals of $5^{\circ}$, until no turbidity was shown by the barium hydroxide solution. In reductions by carbon monoxide, a tendency clearly exists for the carbon dioxide formed to unite with unchanged oxide to form carbonate to some extent, but, with an excess of carbon monoxide passing, this, it is believed, could have but little effect upon the result.

The following shows reduction temperatures for carbon monoxide. (Time of test, six hours.) Silver oxide, $0^{\circ} \mathrm{C}$, ; auric oxide, $\mathrm{O}^{\circ}$; mercurous oxide, $0^{\circ}$; yellow mercuric oxide, $\mathrm{O}^{\circ}$; red mercuric oxide, $95^{\circ}$.

\section{CONCLITSIONS.}

(I) The minimum temperature of reduction of both silver and gold oxide by hydrogen is much below zero, and that for carbon monoxide lower than that for hydrogen.

(2) Carbon monoxide is a more rapid and efficient reducing agent than hydrogen.

(3) The differences in the temperatures of reduction of the yellow and red oxides of mercury make them exhibit properties of two different substances, and confirm the differences previously found in their behavior to chlorine, ${ }^{1}$ oxalic acid, ${ }^{2}$ iodic acid, ${ }^{3}$ and ammonia. ${ }^{4}$ Further work is being done with the oxides of lead and copper.

Polytechnic INSTItute,

BROOKLYN, N. Y.

\section{NOTES.}

Analysis of Water from Hinton, West Virginia.-This water is characterized by a large content of manganese, nickel and cobalt salts. In many mineral waters, manganese salts are reported as present in traces or in comparatively small quantities, but in this water the amount of manganese salts is so large that during evaporation a very marked brown precipitate of a

1 Pelouze : Compt, rend, 16, 50.

2 Millon: Ann. Chem. (Liebig), 18, 349.

3 Rammelsberg: Pogg. Ann, 44, 570.

4 Hirzel: Ann. Chem. (Liebig), 84, 258; Weil: Pogg. Ann., 133, 358. 